1 Radula diversification promotes ecomorph divergence in an adaptive radiation

\title{
of freshwater snails
}

* = Corresponding Author

$\ddagger=$ Shared senior authors

Germany

$143=$ Zoological Research Museum Alexander Koenig, Leibniz Institute for Animal

15 Biodiversity, Bonn, Germany

164 = Naturkundemuseum Potsdam, Potsdam, Germany

$175=$ Centrum für Naturkunde, Hamburg, Germany 


\section{Radula diversification promotes ecomorph divergence in an adaptive radiation}

\section{of freshwater snails}

Abstract

Adaptive diversification of complex traits plays a pivotal role for the evolution of organismal diversity. However, the underlying molecular mechanisms remain largely elusive. In the freshwater snail genus Tylomelania, adaptive radiations were likely promoted by trophic specialization via diversification of their key foraging organ, the radula. To investigate the molecular basis of radula diversification and its contribution to lineage divergence, we use pooled tissue-specific transcriptomes of two sympatric Tylomelania sarasinorum ecomorphs. We show that divergence in both gene expression and coding sequences is stronger between radula transcriptomes compared to mantle and foot transcriptomes. These findings support the hypothesis that diversifying selection on the radula is driving speciation in Tylomelania

34 radiations. We also identify several candidate genes for radula divergence. Putative homologs of some candidates ( $h h, a r x, g b b)$ also contributed to trophic specialization in cichlids and diversification. 


\section{Main}

Adaptive radiations provide extreme examples of rapid phenotypic and ecological diversification and therefore feature prominently among model systems for adaptation and speciation ${ }^{1-6}$. In many adaptive radiations, lineage divergence is promoted by diversification of a few traits, like foraging organs, which acted as key adaptive traits in several radiations , $^{3,7-13}$. Understanding the genetic bases of key adaptive traits is essential because they shape evolutionary trajectories of diversifying lineages ${ }^{14,15}$. Although previous findings are likely biased towards few genes of large effect ${ }^{2,16}$, they also indicate that polygenic selection ${ }^{17-19}$, adaptive introgression ${ }^{20-24}$, and regulatory evolution ${ }^{18,21,25,26}$ promote diversification in adaptive radiations ${ }^{17-19}$. However, much remains to be discovered about the genetic basis of adaptive traits, the molecular evolution underlying their diversification, and their contribution to speciation $^{2,27}$. Particularly, our understanding of gene expression divergence and its contribution to speciation is still in its infancy ${ }^{28,29}$. Here we investigate the genetic basis of diversification of the molluscan key foraging organ (the radula) and its role for lineage divergence in a radiation of freshwater snails, using two sympatric ecomorphs of Tylomelania sarasinorum $^{30}$.

The genus Tylomelania is endemic to the central Indonesian island Sulawesi and underwent several radiations following colonizations of different lake systems ${ }^{31,32}$. Lacustrine species flocks occur across heterogeneous substrates and exhibit remarkable radula diversity (Figure $1)^{33,34}$. In contrast, riverine clades occupy relatively homogenous substrates, have uniformly shaped radular teeth and include comparatively few species ${ }^{33,34}$. Additionally, similar radula morphologies likely evolved several times on similar substrates in different lakes ${ }^{31,32,34}$. Hence, it was hypothesized that divergent adaptation of the radula allowed efficient foraging on alternative substrates and promoted speciation in radiations of Tylomelania ${ }^{31,32}$.

62 In addition to interspecific variation, some species exhibit radula polymorphisms ${ }^{34}$. One such 63 species is Tylomelania sarasinorum, which reportedly has a substrate-correlated radula 64 polymorphism. Ecomorphs occur on rocks and logs in shallow waters of Lake Towuti (Figure 


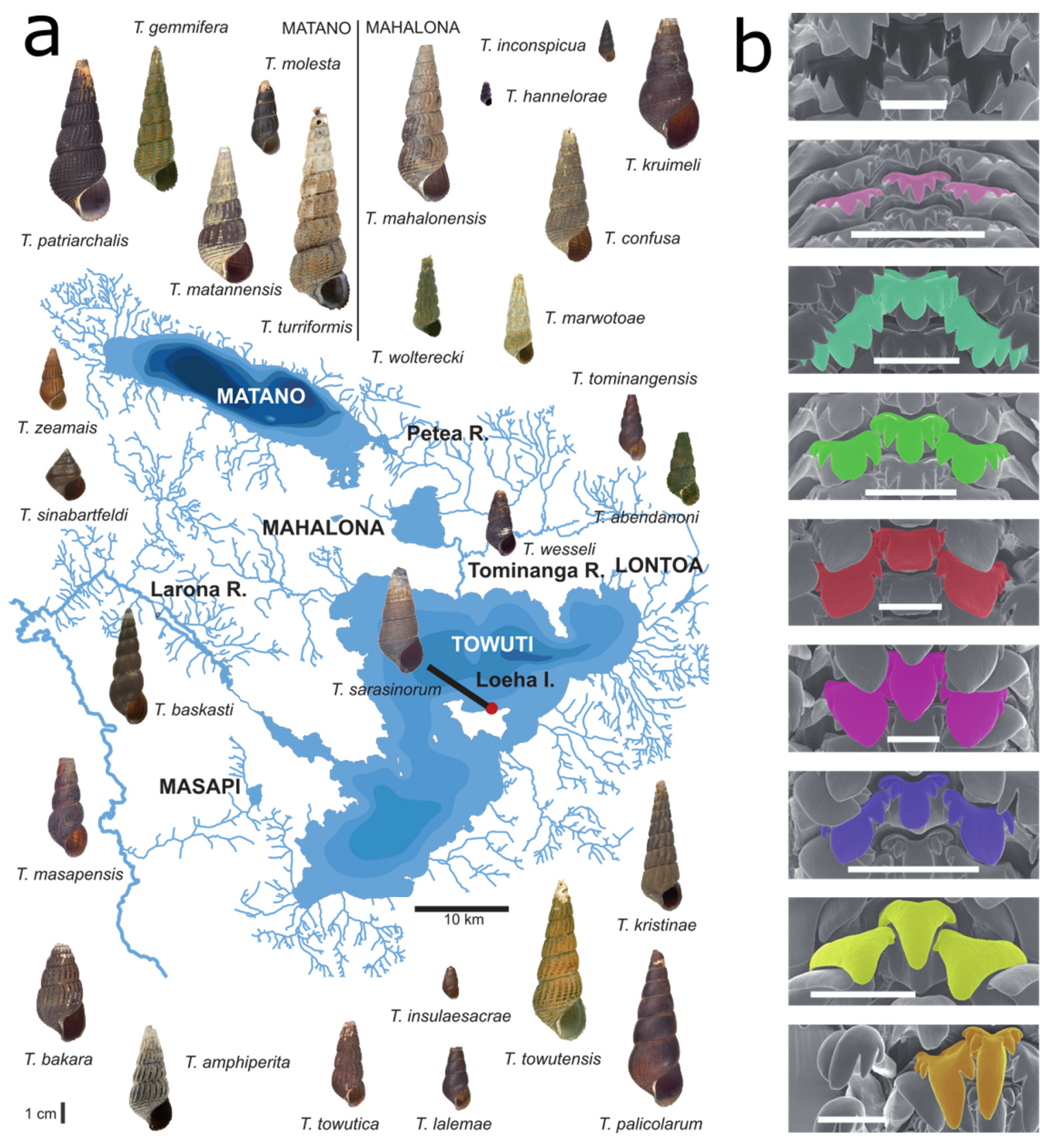

Fig. 1: Diversity of the viviparous freshwater snail genus Tylomelania in the Malili Lakes

System. Species diversity in the Malili Lakes and surrounding rivers (a) is shown together with an overview of radula morphologies $(b)$ (Scale bars $=0.1 \mathrm{~mm}$ ). The sampling site of $T$. sarasinorum specimens at Loeha Island is indicated by a red dot. It was hypothesized that ecomorphs of $T$. sarasinorum modified their radula in adaption to different microhabitats, i.e. submerged logs and rocks. Modified with permission from ${ }^{31}$ and ${ }^{32}$.

$1,2 a)^{34}$, but cannot be distinguished based on mitochondrial markers ${ }^{31,35}$. Given the radula's hypothesized role as key adaptive trait in this radiation, $T$. sarasinorum ecomorphs may represent diverging lineages that evolved different radula morphologies in adaptation to alternative foraging substrates (Figure 2a). Further, the continuous secretion of the radula, 
which consists of numerous rows of chitinous teeth (Suppl. figure 1 ) $^{36}$, enables drastic phenotypic plasticity in some snails ${ }^{37,38}$, showing that tooth shapes can be altered during radula secretion. However, such phenotypic plasticity does not seem to occur in T. sarasinorum. In fact, both ecomorphs can be found across both substrates, yet changes in radula morphology across teeth rows have never been observed in $\sim 500$ specimens (Suppl. table 1).

Here we combine morphological analyses of the radula and the shell with tissue-specific transcriptomes to measure morphological and genetic divergence of sympatric $T$. sarasinorum ecomorphs. Our results indicate evolutionary divergence of ecomorphs. Divergence is most pronounced between radula transcriptomes, adding support to the hypothesis that the radula acts as key adaptive trait in Tylomelania radiations. Putative homologs of candidate genes for radula diversification also contributed to morphological diversification in vertebrate radiations. Our findings indicate that adaptive diversification can leave tissue-specific footprints of transcriptomic divergence, while morphological diversification in adaptive radiations may preferentially be achieved via a limited set of hotspot genes ${ }^{39,40}$ within conserved signaling pathways.

\section{Results and discussion}

\section{Geometric morphometrics corroborates a habitat-correlated radula}

\section{polymorphism}

Although a habitat-correlated radula polymorphism of $T$. sarasinorum together with slight differences in shell morphology and coloration (Figure 2a) have previously been reported ${ }^{41}$, these patterns have not been systematically analyzed so far. Hence, we investigated whether radula morphs are indeed morphologically distinct and whether frequencies of ecomorphs are habitat-correlated at Loeha Island. To this end, we quantified variation in radula and shell morphology of 19 and 18 specimens collected on wood and rock substrates, respectively (See suppl. figure 2 for an overview of radula morphologies). We found that the frequency of both 
radula morphs differs significantly depending on the substrate from which they were collected $(15 / 19=79 \%$ of specimens on rock are rock ecomorph; $18 / 18=100 \%$ of specimens on wood are wood ecomorph; $p=1.01^{*} 10^{-6} ; x^{2}$ test). Furthermore, the first principal component (PC1) of radula shape, which accounts for $93.4 \%$ of the variance within the dataset, clearly separates $T$. sarasinorum ecomorphs $(p<0.001)$. No overlap in PC1 exists between radula morphs, even when individuals collected on opposing substrates where included in the analysis (Figure 2b). In contrast, differences in shell shape are both more subtle and more gradual. Although significant differences in shell shape exist in PC2 $(p<0.001)$, this axis summarizes a relatively
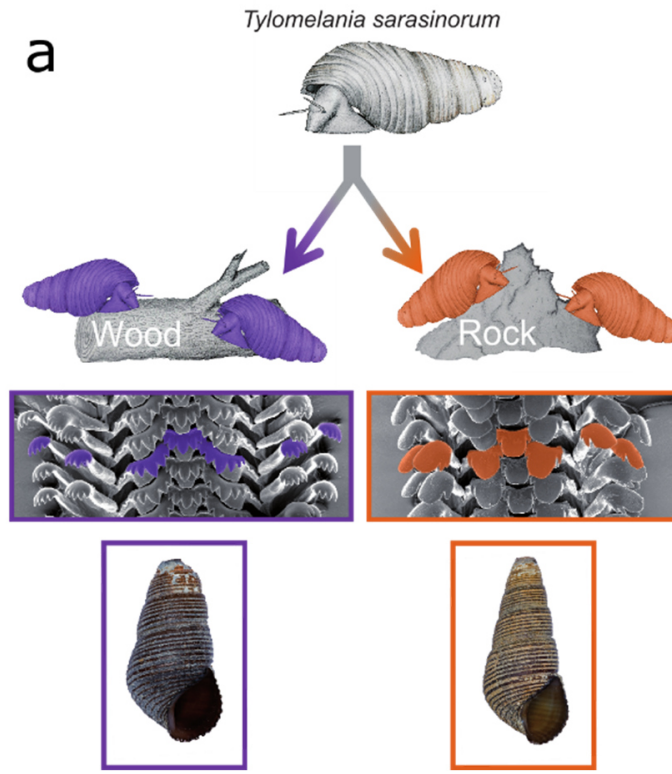
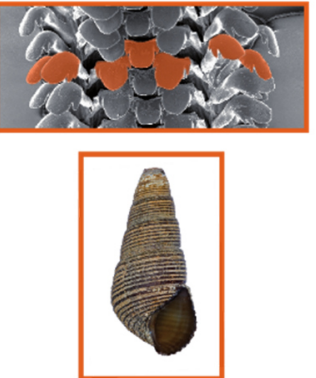

b

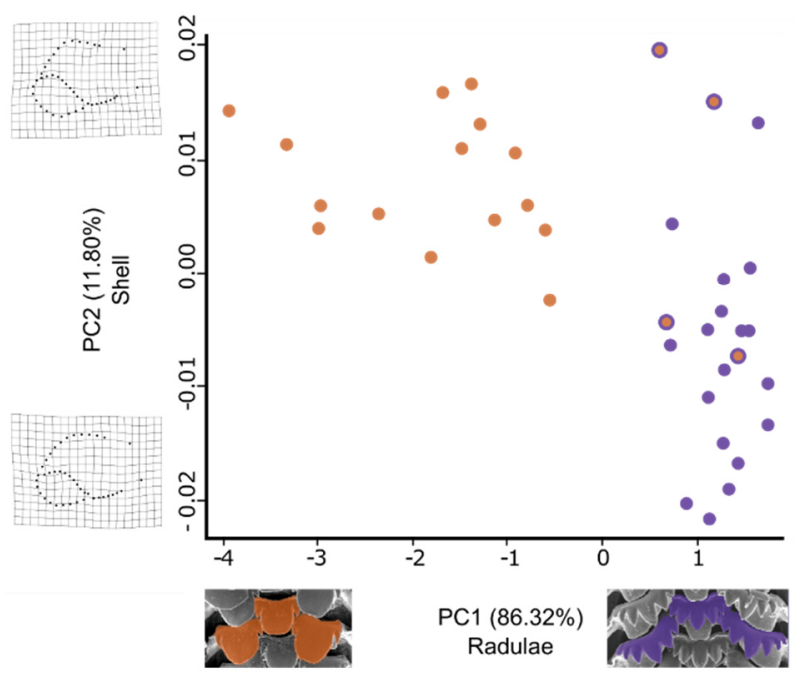

Fig. 2: Habitat-correlated radula polymorphism in $T$. sarasinorum. a) Hypothesis: Radulae of $T$. sarasinorum evolved in adaptation to different microhabitats, i.e. submerged logs and rocks giving rise to diverging ecomorphs. Subtle differences in shell shape and coloration likely also exist. b) Scatterplot based on the two principal components of shell and radula shape that differ significantly between wood (purple) and rock (orange) ecomorphs. Thin plate splines visualize shape change explained by PC2 of shell shape. The center of each dot indicates the habitat from which individuals were collected while the outer ring indicates to which ecomorph it was assigned based on SEM inspection of the radula. Individuals of both ecomorphs are associated with alternative substrates and clearly differ in radula tooth morphology. However, less pronounced, more gradual differences in shell shape also exist.

104 small proportion of shell shape variance (11.8\%) (Suppl. tables 2, 3). Furthermore, shell 105 morphospaces of both ecomorphs are largely overlapping along this axis (Figure 2b). Taken 106 together our data support a habitat-correlated radula polymorphism in $T$. sarasinorum. 
107 Consequently, T. sarasinorum ecomorphs represent a promising model to study the molecular

108 basis of radula disparity and its role for lineage divergence in adaptive radiations of 109 Tylomelania.

\section{Transcriptome sequencing and assembly}

112 To gain insight into transcriptomic divergence of sympatric $T$. sarasinorum ecomorphs, we

113 pooled tissues from four to five individuals and conducted RNAseq on four biological replicates

114 of each mantle, radula formative tissue (Suppl. figure 1) and foot tissue from both ecomorphs.

115 A single transcriptome was assembled from combined data of both ecomorphs. Removal of 116 genes with low expression and clustering of sequences with high sequence similarity (>97\%)

117 reduced the number of contigs with gene status (assigned by Trinity) from 478,661 to 156,685

118 and increased N50 from 613 bp to 1229 bp (Table 1). Importantly, filtering did not affect

119 assembly completeness (89\%) based on a search for 843 conserved metazoan single-copy

120 orthologs using $\mathrm{BUSCO}^{42}$, but decreased the rate of duplicated single copy orthologs from

$1219.4 \%$ to $7.5 \%$ (Table 1). Since N50, completeness and duplication rate are well within the range

122 of recently published mollusk transcriptomes ${ }^{43,44}$, this assembly was used to analyze

123 transcriptomic divergence between ecomorphs of $T$. sarasinorum. Only three out of four

124 biological replicates were used in the subsequent analyses after pool1 of either ecomorph was

125 identified as outlier in initial gene expression analyses (Suppl. figure 3$)^{45}$.

Table 1: Assembly statistics of the raw and filtered assembly

\begin{tabular}{cccccc} 
& $\begin{array}{c}\text { Trinity } \\
\text { genes }\end{array}$ & $\begin{array}{c}\text { GC } \\
\text { (in \%) }\end{array}$ & $\begin{array}{c}\text { 'gene' } \\
\text { N50 }\end{array}$ & $\begin{array}{c}\text { Complete } \\
\text { (in \%) }\end{array}$ & $\begin{array}{c}\text { Duplicated }^{\text {a }} \\
\text { (in \%) }\end{array}$ \\
\cline { 2 - 6 } $\begin{array}{c}\text { Raw } \\
\text { assembly }\end{array}$ & 478661 & 45.2 & 613 & 89 & 9.4 \\
\cline { 2 - 6 } $\begin{array}{c}\text { Filtered } \\
\text { assembly }\end{array}$ & 156685 & 44.9 & 1229 & 89 & 7.5 \\
\cline { 2 - 6 } & & & & &
\end{tabular}

a According to BUSCO 


\section{Transcriptome wide SNP data indicates divergence of $T$. sarasinorum}

\section{7 ecomorphs}

128 Lineage diversification in adaptive radiations of Tylomelania was hypothesized to be promoted by radula diversification in adaptation to alternative substrates ${ }^{31,32}$. Hence, we investigated

130 whether sympatric radula morphs of $T$. sarasinorum with different habitat preferences

131 represent diverging evolutionary lineages. To this end, population genetic analyses were

132 carried out with PoPoolation $2^{46}$ using a uniform coverage of $20 x$ and $10 \%$ minor allele 133 frequency (MAF) for SNP detection. In a total of $39,631,840$ bases that passed the filtering 134 steps, 517,825 putative SNPs could be identified. Of these putative SNPs, 6,366 SNPs (1.2\%) 135 in 2,572 transcripts ( $7.8 \%$ of all transcripts with putative SNPs) qualified as alternatively fixed 136 between ecomorphs $\left(F_{s t}=0\right.$ in all within-morph comparisons and $F_{s t}=1$ in all across-morph 137 comparisons). Figure 3a depicts pairwise genetic differentiation between all pools, while Figure $1383 \mathrm{~b}$ shows the SNP-wise $\mathrm{F}_{\text {st }}$ distribution between both ecomorphs. Although the majority of 139 genetic variation is shared between populations of both radula morphs at Loeha Island (median $140 \quad F_{s t}=0.14 ;$ mean $F_{s t}=0.23$ ), we observed an excess of highly differentiated loci and consistently 141 higher $F_{\text {st }}$ between pools of different ecomorphs (Figure 3). While median $F_{s t}$ in pairwise

a

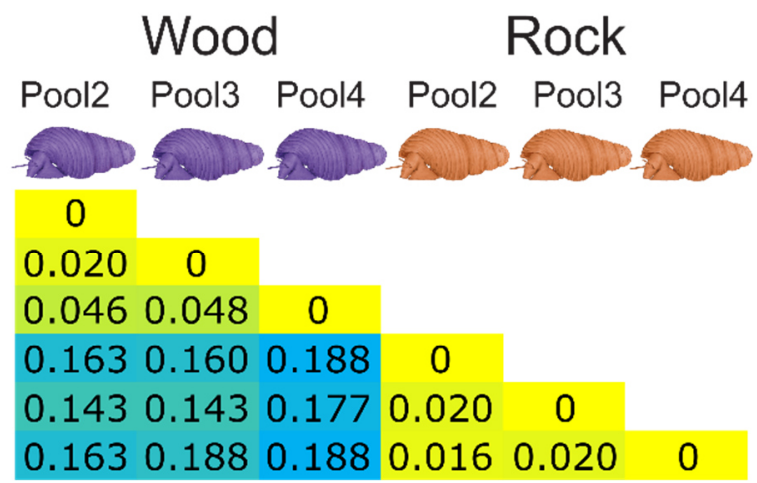

b

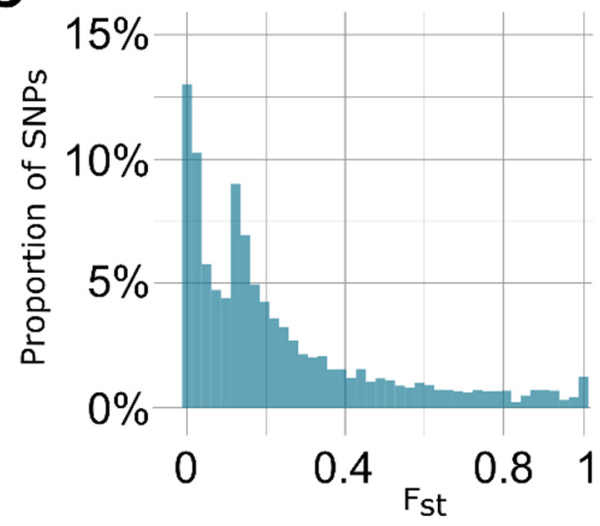

Fig. 3: Divergence of Tylomelania sarasinorum ecomorphs. a) For all pairwise comparisons of pools from wood (violet) and rock (orange), differentiation measured as median SNP-wise $F_{\text {st }}$, is depicted. The degree of differentiation from low to high is indicated by color change from yellow to blue. b) Distribution of SNP wise $F_{\text {st }}$ between different ecomorphs for 517,852 putative SNPs (60x (20x per pool) minimum coverage, 10\% MAF, all pools of each ecomorph combined). While some SNPs exhibit high differentiation, the majority of variation is shared among both ecomorphs. 
142 comparisons among pools of similar ecomorphs ranged from 0.016 to 0.048 , it ranged from

1430.143 to 0.188 among pools of different ecomorphs. Accordingly, our transcriptome-wide SNP

144 data indicate evolutionary divergence of sympatric radula morphs of $T$. sarasinorum at Loeha

145 Island. Although the $F_{\text {st }}$ distribution indicates high differentiation of a few genomic regions in a

146 background of shared genetic variation, pooled transcriptomic data does not allow to reliably

147 distinguish between potential scenarios that may have given rise to this pattern. One possibility

148 that could give rise to such patterns is divergence with gene flow ${ }^{47,48}$. During divergence with

149 gene flow, a few loci under selection become fixed whereas genomic variation at sites of the

150 genome that are not in strong linkage with selected loci are homogenized by gene flow, as

151 Iong as reproductive isolation remains incomplete ${ }^{47,48}$. In fact, individuals with intermediate

152 phenotypes and non-resolving phylogenies from mitochondrial markers indicate that gene flow

153 may not only exist between ecomorphs, but even among $T$. sarasinorum and other species ${ }^{34,49}$.

154 However, other scenarios like divergence without gene flow combined with selective sweeps,

155 potentially following secondary contact, may result in similar patterns, albeit with increased

156 absolute divergence in regions that are not linked to outlier loci. Genomic data comprising

157 individuals from other sites and ideally other species would be required to investigate

158 population history and gene-flow among divergent lineages to decide between alternative 159 explanations.

161 Ecomorphs differ in gene expression across all investigated tissues

162 Regulatory evolution resulting in divergent gene expression plays a key role in adaptation and 163 speciation ${ }^{29,50}$. Although gene expression is known to be highly tissue dependent, our 164 knowledge of tissue-specific transcriptomic divergence is still in its infancy ${ }^{28,51}$. To shed light 165 on gene expression divergence between $T$. sarasinorum ecomorphs, gene expression of foot 166 tissue, shell forming mantle and radula forming tissue of both ecomorphs was analyzed using the pipeline included in Trinity v2.1.152,53. 


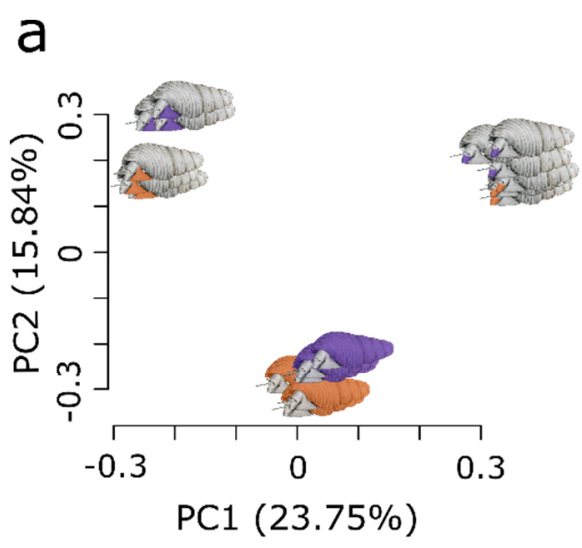

b

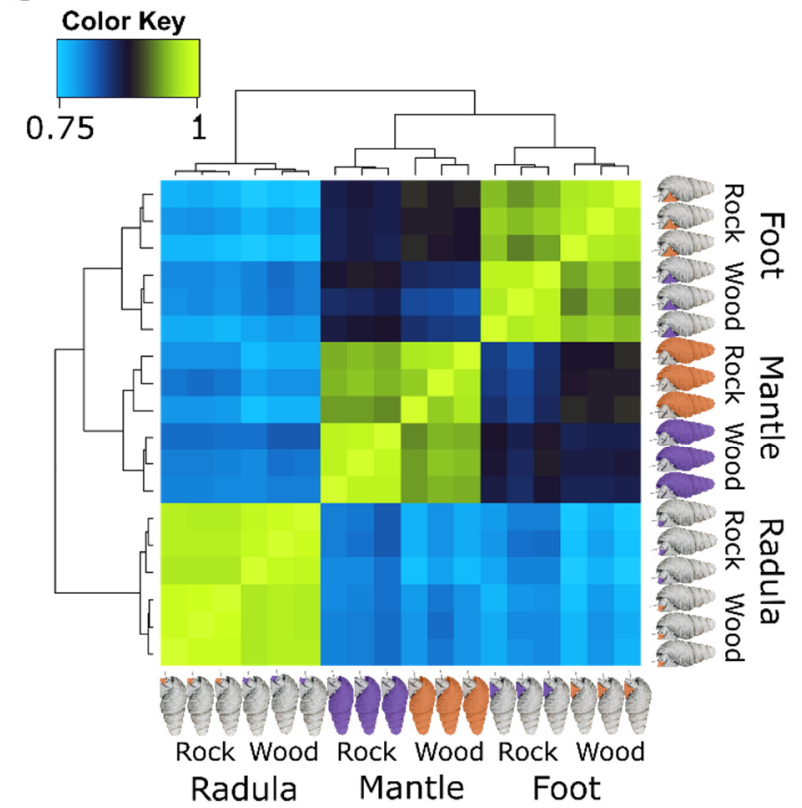

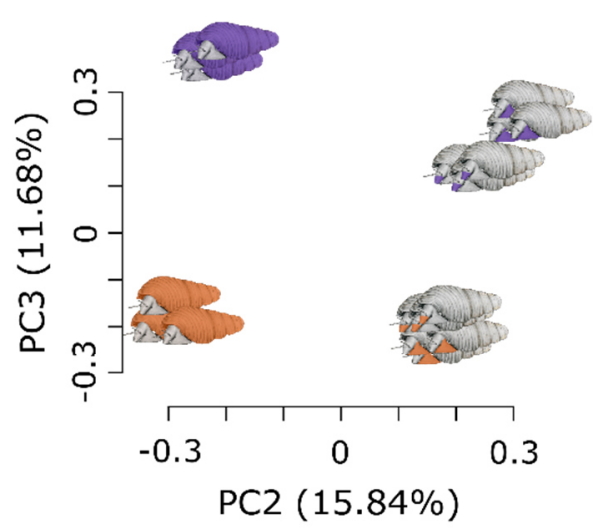

Rock Radula Rock Mantle Rock Foot Wood Radula Wood Mantle Wood Foot

C

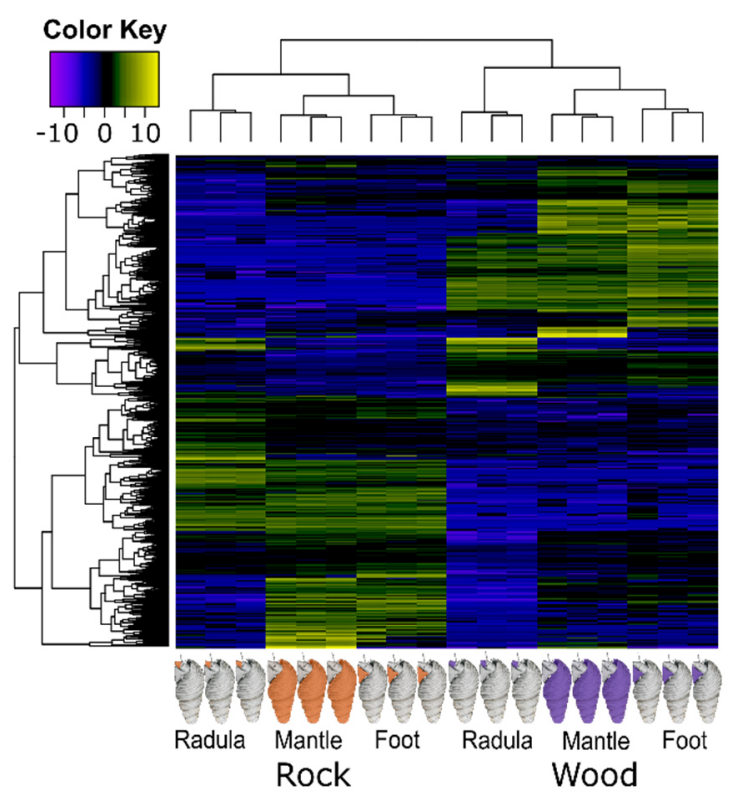

Fig. 4: Divergence of gene expression between $\boldsymbol{T}$. sarasinorum ecomorphs. a) depicts a principal component analysis (PCA) of gene expression in radula, mantle and foot tissue from wood (violet) and rock (orange) ecomorphs. The first and second principal components (PCs) primarily separate different tissue types, while the third PC separates tissues derived from different ecomorphs. b) Hierarchically clustered Spearman correlation matrix of gene expression $\left(\log _{2}\right.$ transformed CPM). Samples with more similar gene expression cluster together in the matrix and the hierarchical clustering tree (left and top) and are colored yellow in the heatmap. c) shows differentially expressed genes between identical tissues of both ecomorphs. Genes are displayed as horizontal lines across samples (columns) in a heatmap of hierarchically clustered, highly differentially expressed ( $p \leq 10^{-10}, F C \geq 4$ ) genes between identical tissues of wood (violet) and rock (orange). Genes with similar expression across samples cluster together in the hierarchical clustering tree on the left, while samples with similar gene expression cluster together in the clustering tree on the top. Overexpressed genes in a sample are colored yellow in the heatmap, while underexpressed genes are displayed in blue. 
170 In accordance with previous work, foot and mantle form sister clusters to the exclusion of the

171 radula cluster and biological replicates cluster tightly together in both PCA and hierarchical

172 clustering, i.e., without a priori assumptions concerning group affiliation (Figure $4 a, b)^{45}$. Within

173 tissues, samples of different ecomorphs form separate clusters, indicating divergence in gene

174 expression across all investigated tissues (Figure 4a, b). Finally, we analyzed genes that are

175 highly differentially expressed between identical tissues of both ecomorphs (false discovery

176 rate $(\mathrm{FDR}) \leq 10^{-10}$; fold change $(\mathrm{FC}) \geq 4$; Figure $\left.4 \mathrm{c}\right)$. Although overall fewer genes are

177 expressed in the radula than in the other two tissues, more genes are highly differentially

178 expressed between the radula forming tissues $(n=536,0.81 \%$ of genes that are expressed in

179 at least one radula tissue) than between mantle $(n=436,0.34 \%)$ or foot tissues $(n=424$,

$180 \quad 0.42 \%$ ) of the two ecomorphs. Stronger morphological disparity in the radula compared to the

181 two other tissues is thus mirrored by more pronounced differences in gene expression, which

182 indicates that regulatory evolution contributes to morphological radula disparity.

183

184 Elevated divergence of radula transcriptomes supports the radula's role as key

185

186

187

188

189

190

191

192

193

194

195

196

\section{adaptive trait}

Selection experiments have shown that strong selection can result in rapid tissue-specific transcriptomic divergence ${ }^{54}$. We thus hypothesized that if diversifying selection on the radula drove divergence of $T$. sarasinorum ecomorphs, divergence of the radula transcriptome would be stronger than transcriptomic divergence of other tissues. To further investigate the contribution of changes in gene regulation and protein coding sequences to radula evolution, we determined tissue-wise transcriptomic divergence in both gene expression and coding sequences.

Divergence in gene expression was measured as the proportion of highly differentially expressed genes $\left(\right.$ FDR $\left.\leq 10^{-10}\right)$ between the same tissue types of both ecomorphs. In this context, genes which are universally differentially expressed across all sampled tissues are uninformative and were excluded from the analysis. We found that divergence in gene 
expression is significantly higher in the radula than in the mantle $\left(97 \%\right.$ higher; $p<10^{-5}$; Fisher's exact test) and in foot $\left(85 \%\right.$ higher; $\left.p<10^{-5}\right)$, while no significant difference exists between the latter two $(6 \%$ higher in foot, $p=0.42)$ (Figure $5 a)$. The radula also has the highest proportion
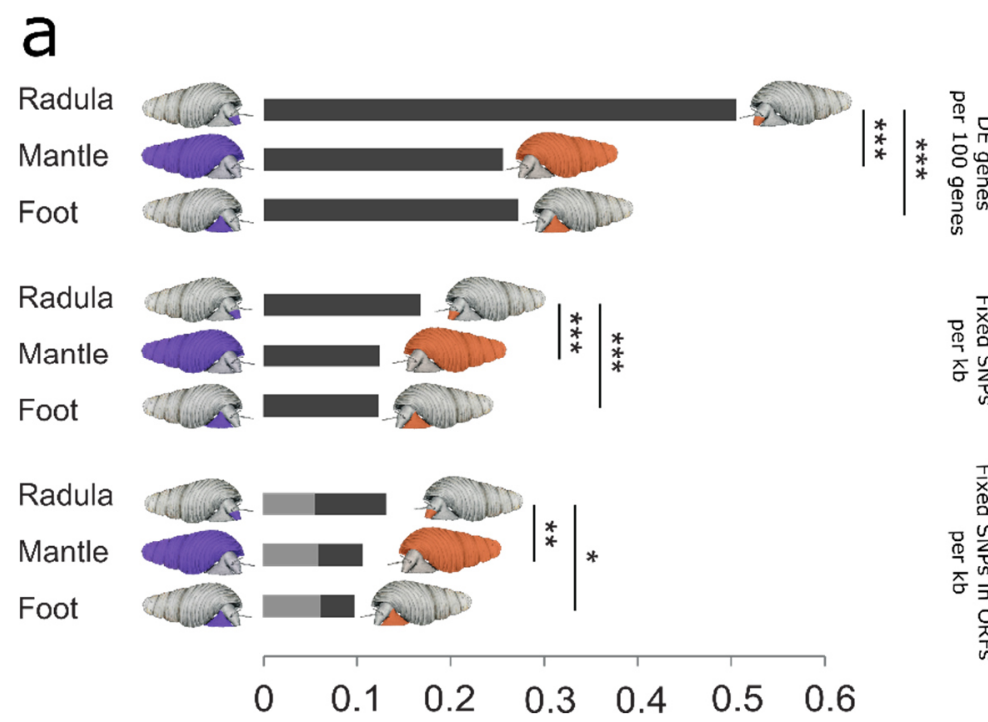

b

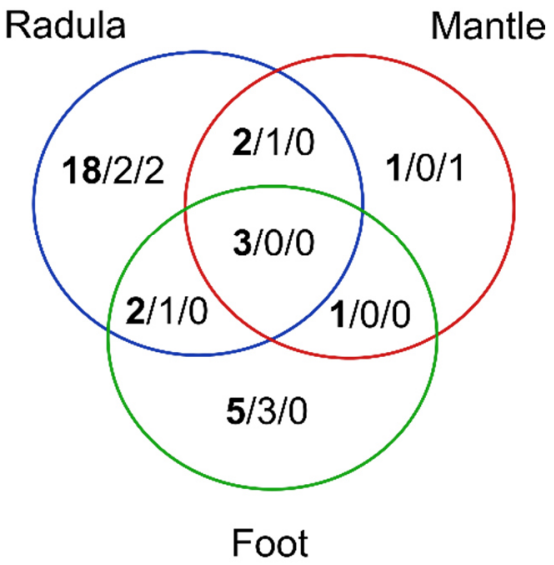

Fig. 5: Tissue-wise transcriptomic divergence of Tylomelania sarasinorum ecomorphs. The proportion of highly differentially expressed (DE) genes between rock and wood ecomorphs, frequency of alternatively fixed SNPs, and frequency of such SNPs in ORFs (black = synonymous; grey = nonsynonymous) is shown for genes expressed in each tissue separately. For the proportion of alternatively fixed SNPs, only genes that are expressed (FPKM $\geq 1$ ) in the respective tissue, but are not expressed across all tissues are included. Similarly, only genes that are not differentially expressed between ecomorphs across all tissues were considered for tissue-wise rates of differentially expressed genes. Significant differences between tissues are indicated by asterisks $\left({ }^{*}=p \leq 0.05 ;{ }^{* *}=p \leq 0.01 ;{ }^{* * *}=p \leq\right.$ 0.001 ). Divergence in both gene sequences and gene expression is significantly higher in genes that are expressed in the radula. b) Venn graph illustrating the position of alternatively fixed SNPs in genes that are also highly differentially expressed between at least one pair of identical tissues of both ecomorphs. The total number of SNPs in highly DE genes is shown first and in bold, followed by the number of synonymous and non-synonymous SNPs in these genes. The majority of alternatively fixed SNPs lie outside of ORFs and are found in genes that are only highly differentially expressed between radula forming tissues. 
Similar to the proportion of differentially expressed genes, the frequency of alternatively fixed SNPs in transcripts of genes that were not expressed across all tissues (FPKM $<1$, i.e. less than one mapped fragment per kilobase of transcript per million mapped reads, in all biological replicates of one tissue) is significantly higher in the radula than in mantle $(\sim 34.4 \%$ higher in radula, $\left.\mathrm{p}<10^{-5}\right)$ or foot $\left(36.6 \%\right.$ higher in radula, $\left.\mathrm{p}<10^{-5}\right)$, but does not differ significantly between the latter two $(1.6 \%$ higher in mantle, $p=0.84)$ (Figure $5 a)$. This pattern remains

211 unchanged when only alternatively fixed SNPs within open reading frames (ORFs) are 212 considered (radula vs mantle: $23.4 \%$ higher in radula, $p=0.036$; radula vs foot: $34.7 \%$ higher 213 in radula, $p=0.005$; mantle vs foot: $9.2 \%$ higher in mantle, $p=0.30$ ). No significant differences 214 among tissues were found when the analysis was restricted to non-synonymous, alternatively 215 fixed SNPs (radula vs. mantle: $6.5 \%$ higher in mantle, $p=0.72$; radula vs foot: $11.3 \%$ higher 216 in foot, $p=0.51$; mantle vs foot: $4.5 \%$ higher in foot, $p=0.65$ ) (Figure $5 a)$. Finally, when both 217 datasets were combined, the majority of genes with alternatively fixed SNPs that were also 218 highly differentially expressed between ecomorphs were only highly differentially expressed 219 between the radula tissues (Figure 5b, but see suppl. figure 4 for lower DE threshold).

220 Our findings indicate that both the rate of highly differentially expressed genes and the 221 frequency of alternatively fixed SNPs are significantly higher in genes expressed in the radula, compared to genes expressed in the other investigated tissues, which is in accordance with stronger diversifying selection on the radula transcriptome. An alternative explanation for a higher rate of differentially expressed genes in the radula would be that the possibility for more precise sampling of radula forming tissue reduces noise in gene expression data, which favors 226 the detection of differentially expressed genes. However, higher sequence divergence of the 227 radula transcriptomes cannot be explained in a similar fashion. Hence, tissue-specific patterns of transcriptomic divergence add support to the hypothesis that diversifying selection on the radula in the course of adaptation to alternative substrates promotes lineage diversification in 230 the adaptive radiations of Tylomelania ${ }^{31}$.

231 Although genes expressed in the radula exhibited significantly higher sequence divergence 232 compared to mantle and foot, no significant differences were detected for non-synonymous 
233 mutations (Figure 5a). Sequence divergence outside of ORFs may reflect divergent non-

234 coding RNAs or untranslated regions of protein coding transcripts, both of which can be linked

235 to post-transcriptional regulation ${ }^{55,56}$. Overall, regulatory evolution appears to dominate

236 divergence of Tylomelania ecomorphs as indicated by highly divergent gene expression across

237 all tissues, which is most pronounced between radulae and higher divergence in untranslated

238 than translated regions, both in general and in transcripts of highly DE genes (Figure 5a,b).

239 These findings are in accordance with the expectation that the relative contribution of protein

240 coding evolution to phenotypic disparity ceases over time, because selection favors regulatory

241 change that can avoid deleterious pleiotropic effects ${ }^{39,40}$. Additionally, our results suggest that

242 divergence of ecomorphs is likely polygenic, which is in line with results from other study

243 systems. For example, regulatory evolution contributed to ecological divergence in East

244 African cichlids, Darwin's finches and sticklebacks ${ }^{2,18,21,57}$, and polygenic selection gave rise

245 to convergent gene expression in lake whitefish radiations in Europe and North America ${ }^{29}$.

\section{Functional enrichment of differentially expressed genes hints at tetrapyrrole}

\section{synthesis underlying shell color disparity}

249 To gain insight into dominant molecular functions (MF), cellular components (CC) and

250 biological processes (BP) in genes contributing to divergence between ecomorphs, transcripts

251 were functionally annotated with the Trinotate annotation pipeline (https://trinotate.github.io/)

252 and gene ontology (GO) enrichment analyses were carried out with $\mathrm{GOseq}^{58}$. Similar to

253 previously published mollusk transcriptomes ${ }^{43,45}$, only a minority of transcripts could be

254 annotated $(n=29.139 ; 19 \%)$ and GO terms were assigned to $13 \%(n=20,864)$ of all

255 sequences in the final assembly. The only enriched GOs among all transcripts with

256 alternatively fixed SNPs were the BP term "biological process" and the CC term "cellular

257 component", indicating that non-synonymous SNPs accumulated in transcripts with unknown

258 functions. In contrast, highly differentially expressed genes between identical tissues of the

259 two ecomorphs were enriched in multiple MF $(n=9)$ including "carbohydrate binding", "heme 
binding" and "tetrapyrrole binding" (Suppl. table 5). The enriched GO-term "carbohydrate binding" is unsurprising within this context, because the radula is primarily made up of the polysaccharide chitin and proteoglycans are important constituents of the molluscan shell 59 . In contrast, implications of the enriched MF "heme binding" and "tetrapyrrole binding" are not as obvious. Interestingly, tetrapyrroles are important molluscan shell pigments ${ }^{60,61}$ and synthesized in the heme pathway ${ }^{62}$. Tetrapyrrole binding genes that were differentially expressed between ecomorphs are primarily expressed in the shell building mantle and foot of the rock morph and mostly differentially expressed between the mantle tissues of both ecomorphs (Suppl. figure 5). Although further investigations targeting color differences between $T$. sarasinorum ecomorphs are needed, enrichment of these functions suggests that differential gene expression in the tetrapyrrole synthesis pathway in the mantle may underlie shell color differences between the ecomorphs.

\section{Candidate genes for radula disparity include cell-cell signaling genes involved}

To investigate individual genes that contributed to radula diversification, two non-overlapping sets of candidate genes were generated based on i) differential expression and ii) nonsynonymous protein coding sequence divergence. Genes that were highly differentially expressed between the radulae of the two ecomorphs (FDR $\leq 10^{-10}$; FC $\geq 4$ ), but not differentially expressed (FDR $\geq 10^{-5} ; \mathrm{FC} \leq 4$ ) between mantles or foot tissues, were chosen as

280 expression-based candidate genes $(n=230)$. The second set of candidate genes $(n=538)$

281 was composed of genes that were expressed in the radula of both ecomorphs and carried 282 alternatively fixed non-synonymous SNPs. To further narrow down the list of candidates, we 283 focused on genes involved in gene regulation and cell-cell signaling, because both regulatory 284 as well as protein coding evolution of these genes may determine when and where radula 285 tooth matrix is secreted. 
While most genes with alternatively fixed non-synonymous SNPs only had one such SNP 287 (66\%), a maximum of 12 such SNPs (and 10 synonymous) was found in Rho GTPase activating protein 21 (rhg21) (Suppl. figure 6). Rho GTPase activating proteins are important activators of Rho family GTPase signaling. Rho family GTPase signaling interacts with notch signaling and regulates various cellular functions, including cytoskeletal reorganization in response to extracellular stimuli ${ }^{63-66}$. Coordinated reorganization of the cytoskeleton is

292 particularly interesting with respect to the radula polymorphism of $T$. sarasinorum, because 293 odontoblasts undergo pronounced shape changes during radula tooth secretion, and 294 modification of their cell shape likely influences tooth morphology ${ }^{36}$. In addition to changing 295 odontoblast cell shapes, modified cytoskeletons may change the localization of chitin synthesis via altered actin filament guidance of a lophotrochozoan-specific chitin synthase with a myosin 297 head $^{67}$ that is expressed in radula forming tissue ${ }^{45}$. The number of non-synonymous 298 alternatively fixed SNPs per kb of ORF in rhg21 corresponds to a 32.2-fold and 2.37-fold 299 increase in frequency of such SNPs compared to the average of all transcripts in the analysis 300 and all transcripts with alternatively fixed SNPs, respectively. Unless mutation rates of genes 301 like rhg21 are substantially increased, their alleles diverged before the divergence of $T$. sarasinorum ecomorphs and either persisted as standing genetic variation, or were introgressed from different lineages. Gene flow among diverging lineages and even

304 introgression from more distantly related species is common in adaptive radiations and may 305 generate and maintain genetic variation at loci underlying adaptive traits $2,18,20,21,23,24,68-72$. Since 306 previous studies indicate abundant hybridization among species of Tylomelania ${ }^{31,34}$, 307 extraordinary divergence of a few genes like rhg21 between T. sarasinorum ecomorphs 308 (Suppl. figure 6) indicates that selection on highly divergent introgressed alleles may also contribute to lineage divergence in adaptive radiations of Tylomelania. Genomic data from across the radiation could be used to test this hypothesis, which, if confirmed, would add further 311 support to a combinatorial view on speciation and adaptive radiation (reviewed in ${ }^{73}$ ).

312 SNP-based candidates further included a transcript that was annotated as neurogenic notch 313 locus 1 (notch1) and a transcript encoding strawberry notch (1 non-syn; 5 syn), whose role in 
314 the notch signaling pathway is still unclear ${ }^{74}$. A putative homolog of notch1 was also found

315 among the expression-based candidate genes, together with the morphogen hedgehog ( $h h)$.

316 Both the notch and the hedgehog signaling pathway are conserved across the bilaterians and

317 interact during developmental tissue patterning ${ }^{75,76}$. Interestingly, the hedgehog signaling

318 pathway mediates both fixed as well as phenotypically plastic effects on jaw morphology in

319 East African cichlids ${ }^{77-79}$. Hedgehog $(h h)$ further regulates bone morphogenetic protein (BMP)

320 expression in several metazoan lineages ${ }^{76,80,81}$ and regulatory evolution resulting in divergent

321 expression of BMPs played a pivotal role for craniofacial diversity in both Darwin's finches and

322 East African cichlids ${ }^{26,27,82,83}$. In $T$. sarasinorum, hedgehog $(h h)$ is overexpressed in the radula

323 of the rock morph and a BMP that is most similar to $g b b / B M P 5-8$ is only expressed (FPKM $\geq$

324 1) in the radula of the rock morph. The only homeobox gene found among candidate genes was annotated as aristaless related homeobox protein (arx) (4 non-syn; 2 syn). In our dataset, arx is only expressed in the radula tissue and carries four non-synonymous alternatively fixed

327 SNPs. Similar to notch signaling, we previously found that arx likely plays an important role for 328 radula formation ${ }^{45}$. Interestingly, two non-synonymous substitutions in the aristaless-like homeobox 1 transcription factor (ALX1), which has an important role for craniofacial development in vertebrates, promoted beak diversification in Darwin's finches ${ }^{21}$.

331 In summary, we find that several close relatives and putative homologs of genes that 332 contributed to diversification of beaks in Darwin's finches and/or yaws of East African cichlids 333 might also be involved in the adaptive diversification of the radula. Although, given similar gene 334 regulatory networks, evolutionarily relevant mutations are expected to accumulate in so-called 335 hotspot genes ${ }^{39,40}$, the radula does not share the developmental basis that jaws and beaks 336 have in common ${ }^{84}$. Nonetheless, our observations might be explained by a relatively restricted 337 and highly conserved set of tissue patterning cell-cell signaling pathways ${ }^{85}$ that contain a 338 limited set of genes that have the potential to rapidly generate potentially adaptive 339 morphological diversity without fatal pleiotropic effects ${ }^{39,40,76,86}$. While a large number of 340 candidate genes in this study calls for further verification, our results indicate that diversification 
341 of foraging organs in adaptive radiations might be achieved via a limited set of cell-cell

342 signaling genes that are particularly prone to rapid adaptive diversification.

\section{Conclusions}

346 This study confirms habitat-correlated radula disparity in T. sarasinorum, shows evolutionary

347 divergence of ecomorphs and corroborates the hypothesis that adaptive diversification of the 348 radula drives lineage divergence in adaptive radiations of Tylomelania. Exceptional sequence 349 divergence of some genes may be a sign of older, potentially introgressed, variation, but needs 350 to be further investigated using population genomic data from other sites and including related 351 species. More generally, our findings shed light on tissue-wise transcriptomic divergence and 352 indicate that adaptive diversification can leave tissue-specific footprints. Finally, overlapping 353 gene sets appear to underlie rapid adaptive diversification of foraging organs in radiations of 354 fishes, birds and snails, which is an aspect that requires further investigation in the future to 355 get a better understanding of the genetic mechanisms generating functional diversity within 356 adaptive radiations.

\section{Materials and Methods}

\section{Specimen and tissue collection}

361 Adult specimens of Tylomelania sarasinorum were collected from submerged wood and rock substrates at the northern shore of Loeha Island (Lake Towuti, South Sulawesi, Indonesia;

$3632.76075 \mathrm{~S} 121.5586 \mathrm{E})$. All snails were collected in close proximity to each other and kept in 364 buckets filled with lake water for a few hours before they were dissected in the field. Tissue 365 samples of radula forming tissue, mantle edge, and foot muscle were directly stored in 366 RNAlater to ensure RNA preservation. Before any samples were pooled for RNA extractions, 
radula forming tissue was separated from the rest of the radula (Suppl. figure 1) and radula morphs of all individuals were inspected using scanning electron microscopy.

\section{Morphological analyses}

371 Shell shape and radula meristic were assessed for a total of 37 adult specimens from the

372 collection of the Natural History Museum Berlin (Suppl. figures 1,7). Specimens were chosen

373 from lots that had been sampled randomly by hand from wooden $(n=19)$ and rocky substrate

$374 \quad(n=18)$.

375 Variation in shell shape was quantified using landmark based geometric morphometrics

376 methods. To this end, specimens were placed on sand-filled trays and photographed with the 377 aperture facing upwards using a SatScan collection scanner (SmartDrive Limited). Eight 378 landmarks were placed on the whorls and aperture (see Suppl. figure 7a for details). Round 379 structures of the aperture and the first and second whorl were outlined by four sliding 380 semilandmarks (Suppl. figure 7a). Landmarks were placed using the software tpsDIG2 ${ }^{87}$.

381 Differences in size and rotation were removed from the data with a Procrustes superimposition

382 (gpagen function in the geomorph package ${ }^{88}$ ). A principal component analysis (PCA) was 383 calculated on the Procrustes residuals using the plotTangentSpace function ( $r$ package 384 geomorph ${ }^{88}$ ). T-tests were calculated for principle components that explained more than $5 \%$ 385 of the total variance, to test for significant differences in shell shape between ecomorphs.

386 Radulae were dissected from the headfoot of the animals and surrounding tissue was digested 387 with $500 \mu$ lysis buffer ${ }^{89}$ and $10 \mu l$ proteinase $\mathrm{K}$ at $55^{\circ} \mathrm{C}$ overnight. Afterwards, radulae were 388 cleaned with ethanol and treated for 2 seconds in an ultrasound bath. Radulae were mounted 389 on electron microscope stubs and sputter coating was carried out with the Quorum Q150RS 390 Sputter Coater using the manufacturer's program number 2.

391 The number of teeth was counted and maximum width and total height of the central denticle 392 as well as the total width of the rachis base were measured with the software Image ${ }^{90}$ (Suppl. 393 figure 7b). Subsequently, ratios of central denticle width/total height and rachis width were calculated. A PCA was carried out with these ratios and the number of denticles of the rachis 
395 (the central tooth). Two tailed t-tests were used to evaluate morphological differences between

396 ecomorphs for each PC that explained more than $5 \%$ of the total variance.

\section{Sample preparation and sequencing}

399 Nineteen individuals of the $T$. sarasinorum wood morph were grouped into three pools of five 400 and one pool of four individuals (data already used $\mathrm{in}^{45}$ ), and 20 individuals of the $T$. 401 sarasinorum rock morph were grouped into four pools of five individuals. Tissue samples of 402 individuals in each pool were weighed (Mettler AT 261 scale), and similar amounts of each 403 individual were pooled, resulting in four biological replicates of each tissue. Tissue was 404 homogenized with a Precellys Minilys, and total RNA was extracted using two alternative protocols. Since larger amounts of foot tissue were available, RNA was extracted from foot 406 muscle with a TRIzol囚 extraction according to the manufacturer's protocol. However, to extract 407 RNA from minute amounts of radula formative tissue and mantle edge, a customized protocol 408 of the RNeasy Plus Micro Kit (Qiagen) was employed ${ }^{45}$. Briefly, remaining tissue fragments 409 were digested with proteinase $\mathrm{K}$ following mechanical homogenization. Subsequently, lysis 410 buffer was added to allow efficient DNA removal with gDNA spin columns. Amount and quality 411 of extracted total RNA was inspected using Agilent's 2100 Bioanalyzer. Tylomelania 412 sarasinorum rRNA carries a "hidden break", which means that the 28S rRNA easily 413 disintegrates into two smaller fragments. This led to a sharp $18 \mathrm{~S}$ band, but a much reduced or 414 lacking 28S rRNA peak in our samples. Hence, RNA integrity (RIN) estimates were not 415 applicable. Nonetheless, samples showed no signs of degradation or DNA contamination. 416 Messenger RNA was enriched with poly (A) capture using NEXTflex TM Poly $(A)$ Beads, and 417 strand-specific libraries were built using the NEXTflex ${ }^{\mathrm{TM}}$ Rapid Illumina Directional RNA-Seq 418 Library Prep Kit (Bioo Scientific) with modifications suggested by Sultan et al. (2012). Quality 419 and concentrations of libraries were evaluated using Agilent's 2100 Bioanalyzer and qPCR 420 (Kapa qPCR High Sensitivity Kit). Libraries had average fragment sizes between 350-500 bp 421 and were sequenced (150 bp, paired end) on an Illumina NextSeq sequencing platform at the 422 Berlin Center for Genomics in Biodiversity research (BeGenDiv). 


\section{Transcriptome assembly}

425 Raw sequences were trimmed with a quality threshold of 30 , minimum read length of $25 \mathrm{bp}$, and all Ns were removed using sickle ${ }^{92}$. Adapter sequences were subsequently removed with cutadapt ${ }^{93}$, which generated a final dataset consisting of 941 million paired end reads (Suppl. table 5). Trinity v2.1.152,53 was run in strand-specific mode with a minimal transcript length of $250 \mathrm{bp}$, in silico read normalization (max. read coverage $=50$ ), and two-fold minimal kmer coverage to generate a single assembly of all tissues of both ecomorphs. Quality-filtered adapter trimmed reads of each sample were mapped to the transcriptome using bowtie $2^{94}$, followed by abundance estimation with $\mathrm{RSEM}^{95}$. Since abundance of rRNA mostly reflects polyA capture success, ribosomal RNA (rRNA) was removed following identification with a BLAST search using 28S rRNA (Brotia pagodula; HM229688.1) and 18S rRNA (Stenomelania crenulata; AB920318.1) as query sequences. Pool1 mantle and pool1 radula of both ecomorphs were removed from further analyses after they were identified as outliers in 437 principal component analysis (PCA) of $\log _{2}$ transformed counts per million mapped reads 438 (cpm) (Suppl. figure 3). The cause for this observation is likely a combination of lower yield of total RNA in the first extractions, which led to a decrease in library complexity, and deeper

440 sequencing of pool1 (Suppl. table 5). A batch effect might also have contributed to this 441 observation, because pool1 mantle and pool1 radula of both ecomorphs were sequenced 442 separately from all other samples. The assembly was subsequently filtered by expression 443 (FPKM $\geq 1$, i.e. at least one mapped fragment per kilobase of transcript per million mapped 444 reads), using a script provided in the Trinity pipeline. CD-HIT version $4.6^{96}$ was used to cluster 445 the longest isoforms of all "trinity genes" based on sequence similarity (97\% sequence identity 446 threshold; $90 \%$ minimum alignment coverage of the shorter sequence), and the longest 447 transcript of each cluster was retained. Quality filtered, adapter trimmed reads of both 448 ecomorphs were re-mapped to the remaining transcripts, and transcripts with very low 449 expression (FPKM $\leq 1)$ were removed to create a final assembly. BUSCO v1.1b142, was 450 employed to generate estimates of transcriptome completeness, redundancy, and 
451 fragmentation by searching for 843 known metazoan single copy orthologs. Since BUSCO 452 indicated that transcriptome completeness was not negatively affected by the abovementioned 453 filtering steps, the final assembly was chosen for further analyses.

455 Gene expression analysis

456 Gene expression analysis was performed using the pipeline included in Trinity v2.1.152,53. 457 Briefly, quality-filtered adapter-trimmed reads of each sample were mapped to the final 458 assembly using bowtie ${ }^{94}$, followed by abundance estimation with RSEM ${ }^{95}$. Differentially 459 expressed genes (FDR $\leq 10^{-5} ; \mathrm{FC} \geq 4$ ) and highly differentially expressed genes (FDR $\leq 10^{-10}$; $460 \quad \mathrm{FC} \geq 4$ ) were determined for all pairwise morph and tissue comparisons using edge $\mathrm{R}^{97}$.

\section{Annotation}

Transcripts in the final assembly were functionally annotated using the Trinotate annotation pipeline (v3.0.1). Results were imported into the Trinotate-SQLite database, and the annotation report was generated using default parameters. The identities of $T$. sarasinorum genes that are mentioned by name in this manuscript were further varified by searching proteins matching $T$. sarasinorum open reading frames in the UniProt database using BLASTX and manually inspecting alignments of the 10 best hits with an E-value of $10^{-10}$ or lower for which the alignment covered at least $60 \%$ of the database sequence.

\section{Ecomorph divergence}

PoPoolation $2^{46}$ was used to gain insight into divergence of $T$. sarasinorum ecomorphs.

473 Duplicate reads, reads that did not map as proper pairs, and low quality alignments (mapping 474 quality < 20) were removed from mappings using SAMtools v1.398 and Picard Tools 475 (http://broadinstitute.github.io/picard/). Subsequently, mappings of different tissues of the 476 same pool (same individuals) were merged. To reduce biases in SNP detection caused by 477 variance in gene expression, a uniform coverage of $20 x$ for each pool was generated by 
478 subsampling mapped reads (without replacement) and removing all sites with a coverage

$479<20 x$. SNPs were called at a minor allele frequency (MAF) of $10 \%$, i.e. 12 calls in a total of 120

480 calls per site across all six pools. SNPs with lower MAF were discarded to remove potential 481 sequencing errors and uninformative $\mathrm{SNPs}^{99}$, which increases the accuracy of allele frequency 482 estimations ${ }^{100}$. SNP-wise $\mathrm{F}_{\text {st }}$ was calculated for all pairwise comparisons between pools using 483 PoPoolation2 ${ }^{46}$. Median pairwise $F_{\text {st }}$ were estimated from all SNPs for each pairwise 484 comparisons of pools. Median Fst and SNP wise $F_{\text {st }}$ distributions between ecomorphs were 485 calculated based on combined pool-wise allele counts for which a sync file with combined 486 allele counts for each the rock and the wood morph were generated. This resulted in a 487 coverage of $60 \times$ ( 3 pools $\times 20 \times$ coverage per ecomorph). MAF was retained at $10 \%$. 488 Synonymous and non-synonymous mutations were determined using the syn-nonsyn-atposition.pl script included in PoPoolation v1.2.2 based on the longest ORF per gene and using

490 a merged mapping file combining read mappings of all pools. Although PoPoolation is not 491 recommended for processing pooled transcriptome data, because expression differences 492 between individuals and alleles may introduce additional variation compared to sequencing 493 pooled DNA (Pool-Seq) ${ }^{46,100}$, similar approaches have successfully been employed in 494 numerous studies ${ }^{54,101-104}$. Additionally, high repeatability among biological replicates in this study supports the validity of our approach.

\section{Estimating tissue-specific transcriptomic divergence}

498 To evaluate whether transcriptomic divergence between ecomorphs differed depending on the 499 tissue, tissue-wise divergence in gene expression and coding sequences was determined. The proportion of genes that were highly differentially expressed between identical tissues of the ecomorphs (FDR $\leq 10^{-10} ; \mathrm{FC} \geq 4$ ) was calculated for each tissue. Genes that were highly

502 differentially expressed between ecomorphs across all tissues were excluded from the 503 analyses. Likewise, the frequency of alternatively fixed SNPs was determined for genes 504 expressed (FPKM $\geq 1)$ in each tissue, excluding genes that were expressed across all tissues. 505 Genes were regarded as expressed in a certain tissue when they were expressed $(F P K M \geq 1)$ 
506 in at least one pool of that tissue. Differences in the proportion of differentially expressed genes

507 and in the frequency of alternatively fixed SNPs between tissues were evaluated using Fisher's

508 exact test.

510 Gene ontology enrichment

511 Gene ontology (GO) enrichment analyses were carried out to determine dominant functions of

512 genes with alternatively fixed non-synonymous SNPs and of all genes that were highly 513 differentially expressed (FDR $\leq 10^{-10} ; \mathrm{FC} \geq 4$ ) between identical tissues of the two ecomorphs. 514 For all transcripts in the final assembly, GO assignments and parental terms were extracted 515 from the Trinotate annotation report using the script included in the Trinotate-2.0.2 pipeline. 516 GOseq $^{58}$ was used to identify enriched GOs in genes that were differentially expressed 517 between the same tissues of different ecomorphs against a background of all genes in the final 518 assembly. Additionally, enriched gene ontologies were identified in genes with alternatively 519 fixed non-synonymous SNPs against a background of all genes that had bases that passed 520 the filtering for coverage in the PoPoolation pipeline. Significantly enriched gene ontologies 521 with a false discovery rate FDR $\leq 0.05$ were summarized and redundant terms were removed 522 (allowed similarity: 0.5) with REVIGO ${ }^{105}$.

\section{Identification of candidate genes}

525 Alternatively fixed non-synonymous SNPs were used to identify candidate genes for adaptive 526 divergence. Thresholds for outlier detection are always to some extent arbitrary and depend 527 on the choice of MAF that is accepted as informative to detect patterns of selection ${ }^{99,103}$. In 528 addition to demography and stronger purifying selection in the transcriptome resulting in 529 different effective mutation rates ${ }^{106}$, core assumptions of models employed for pooled genomic 530 data may be violated by a larger margin of error in allele frequency estimation from pooled 531 RNA compared to pooled DNA due to variation in gene expression between individuals and 532 even alleles ${ }^{100}$. Accordingly, previous studies based on pooled transcriptomic data mostly used 
533 quantile based approaches for outlier detection ${ }^{101,103}$. We used the most conservative 534 approach available to us and only chose alternatively fixed SNPs, i.e. SNPs with $\mathrm{F}_{\mathrm{st}}=0$ in all 535 within-morph comparisons and $F_{\mathrm{st}}=1$ in all across-morph comparisons (98.8\% percentile). 536 Genes that carried non-synonymous alternatively fixed SNPs and were expressed in both radula forming tissues were determined as candidate genes for radula divergence. Finally, 538 genes that were highly differentially expressed between the radulae of the two ecomorphs 539 (FDR $\left.\leq 10^{-10} ; \mathrm{FC} \geq 4\right)$, but not differentially expressed (FDR $\left.\geq 10^{-5} ; \mathrm{FC} \leq 4\right)$ between mantles 540 or foot tissues, were collected as candidate genes for radula shape divergence.

\section{Data availability}

544 Sequence data and additional information are available at the NCBI Sequence Read Archive 545 (SRP134819, \#\#) and BioProject (BioProject ID: PRJNA437798; BioSample accessions: 546 SAMN08685289 - SAMN08685300 and SAMN13841508 - SAMN13841519).

\section{Acknowledgements}

549 We thank Isabelle Waurick and the BeGenDiv for assistance in the lab, and all members of the 550 Hofreiter Lab, von Rintelen Lab and David Garfield for helpful discussions. LIPI (Indonesian 551 Institute of Sciences) and RISTEK (Indonesian State Ministry of Research and Technology) 552 kindly issued the permits to conduct research in Indonesia. We would further like to thank 553 Ristiyanti M. Marwoto (Museum Zoologicum Bogoriense, LIPI, Cibinong) for her continuous 554 support of the project. This work was financed by the German Research Council (DFG) (grant number: Ri 1738/9-1). The authors declare no conflict of Interest. 


\section{References}

5601 1. Seehausen, O. African cichlid fish: a model system in adaptive radiation research. Proc. $R$.

$561 \quad$ Soc. B Biol. Sci. 273, 1987-1998 (2006).

562 2. Berner, D. \& Salzburger, W. The genomics of organismal diversification illuminated by adaptive 563 radiations. Trends Genet. 31, 491-499 (2015).

564 3. Grant, P. R. \& Grant, B. R. Evolution of character displacement in Darwin's finches. Science.

$565 \quad 313,224-226(2006)$.

566 4. Schluter, D. The Ecology of Adaptive Radiation. (Oxford University Press, 2000).

5. Darwin, C. On the Origin of the Species by Means of Natural Selection: Or, The Preservation of Favoured Races in the Struggle for Life. (John Murray, 1859).

6. Baldwin, B. G. \& Sanderson, M. J. Age and rate of diversification of the Hawaiian silversword alliance (Compositae). Proc. Natl. Acad. Sci. U. S. A. 95, 9402-9406 (1998).

7. Pfaender, J., Schliewen, U. K. \& Herder, F. Phenotypic traits meet patterns of resource use in the radiation of "sharpfin"' sailfin silverside fish in Lake Matano. Evol. Ecol. 24, 957-974 (2010).

8. Martin, C. H., Erickson, P. A. \& Miller, C. T. The genetic architecture of novel trophic specialists: higher effect sizes are associated with exceptional oral jaw diversification in a pupfish adaptive radiation. Mol. Ecol. 26, 624-638 (2017).

9. Nagao, Y. et al. Distinct interactions of Sox5 and Sox10 in fate specification of pigment cells in medaka and zebrafish. PLOS Genet. 14, e1007260 (2018).

10. Elmer, K. R. et al. Parallel evolution of Nicaraguan crater lake cichlid fishes via non-parallel

11. Kocher, T. D. Adaptive evolution and explosive speciation: The cichlid fish model. Nat. Rev. Genet. 5, 288-298 (2004).

12. Pfaender, J., Hadiaty, R. K., Schliewen, U. K. \& Herder, F. Rugged adaptive landscapes shape a complex, sympatric radiation. Proc. R. Soc. London B 283, 20152342 (2016). radiations of birds: comparative morphometrics of Darwin's finches and Hawaiian honeycreepers. Philos. Trans. R. Soc. B Biol. Sci. 372, 20150481 (2017).

14. Ferris, K. G., Barnett, L. L., Blackman, B. K. \& Willis, J. H. The genetic architecture of local adaptation and reproductive isolation in sympatry within the Mimulus guttatus species complex. Mol. Ecol. 26, 208-224 (2016). 
species pairs. Mol. Biol. Evol. 24, 1423-1438 (2007).

16. Rogers, S. M., Xu, S. \& Schlüter, P. M. Introduction: integrative molecular ecology is rapidly advancing the study of adaptation and speciation. Mol. Ecol. 26, 1-6 (2017).

17. Salzburger, W. Understanding explosive diversification through cichlid fish genomics. Nat. Rev. Genet. 19, 705-717 (2018).

18. Brawand, D. et al. The genomic substrate for adaptive radiation in African cichlid fish. Nature 513, 375-381 (2014).

19. Dennenmoser, S., Vamosi, S. M., Nolte, A. W. \& Rogers, S. M. Adaptive genomic divergence under high gene flow between freshwater and brackish-water ecotypes of prickly sculpin (Cottus asper) revealed by Pool-Seq. Mol. Ecol. 26, 25-42 (2017).

20. Richards, E. \& Martin, C. Adaptive introgression from distant Caribbean islands contributed to

21. Almén, M. S. et al. Adaptive radiation of Darwin's finches revisited using whole genome the diversification of a microendemic radiation of trophic specialist pupfishes. PLoS Genet. 13,

22. Dasmahapatra, K. K. et al. Butterfly genome reveals promiscuous exchange of mimicry

23. Meier, J. I. et al. Ancient hybridization fuels rapid cichlid fish adaptive radiations. Nat. Commun.

$$
\text { 8, } 14363 \text { (2017). }
$$

24. Seehausen, O. Hybridization and adaptive radiation. Trends Ecol. Evol. 19, 198-207 (2004).

25. Abzhanov, A. et al. The calmodulin pathway and evolution of elongated beak morphology in Darwin's finches. Nature 442, 563-567 (2006).

26. Abzhanov, A., Protas, M., Grant, B. R., Grant, P. R. \& Tabin, C. J. Bmp4 and morphological variation of beaks in Darwin's finches. Science 305, 1462-1465 (2004).

27. Lawson, L. P. \& Petren, K. The adaptive genomic landscape of beak morphology in Darwin's finches. Mol. Ecol. 26, 4978-4989 (2017).

28. Uebbing, S. et al. Divergence in gene expression within and between two closely related flycatcher species. Mol. Ecol. 25, 2015-2028 (2016). drives the evolution of convergent transcriptomic landscapes across continents within a Nearctic sister-species complex. Mol. Ecol. mec.15226 (2019).

624 30. Kruimel, J. H. Verzeichnis der von Herrn E.C.Abendanon in Celebes gesammelten Süsswasser-Mollusken. Bijdr. tot Dierkd. 19, 217-235 (1913). 
specialization drive adaptive radiation of freshwater gastropods in ancient lakes on Sulawesi, Indonesia. Proc. R. Soc. London B 271, 2541-2549 (2004).

32. von Rintelen, T., von Rintelen, K. \& Glaubrecht, M. The species flocks of the viviparous freshwater gastropod Tylomelania (Mollusca: Cerithioidea: Pachychilidae) in the ancient lakes

33. von Rintelen, T. \& Glaubrecht, M. Anatomy of an adaptive radiation: a unique reproductive of Sulawesi, Indonesia: The role of geography, trophic morphology and color as driving forces

34. Glaubrecht, M. \& von Rintelen, T. The species flocks of lacustrine gastropods: Tylomelania on

35. Hilgers, L., Grau, J. H., Pfaender, J. \& von Rintelen, T. The complete mitochondrial genome of

36. Mackenstedt, U. \& Märkel, K. Experimental and comparative morphology of radula renewal in the viviparous freshwater snail Tylomelania sarasinorum (Caenogastropoda: Cerithioidea).

37. Padilla, D. K. Inducible phenotypic plasticity of the radula in Lacuna (Gastropoda: Littorinidae). Veliger 4, 201-204 (1998).

38. Jensen, K. R. Morphological adaptations and plasticity of radular teeth of the Sacoglossa (= Ascoglossa) (Mollusca: Opisthobranchia) in relation to their food plants. Biol. J. Linn. Soc. 48,

39. Stern, D. L. \& Orgogozo, V. The loci of evolution: How predictable is genetic evolution?

40. Stern, D. L. \& Orgogozo, V. Is genetic evolution predictable? Science 323, 746-751 (2009). morphological review of an endemic species flock of Tylomelania (Gastropoda: Cerithioidea: Pachychilidae) in the Malili lake system on Sulawesi, Indonesia. Hydrobiologia 592, 11-94 (2007).

42. Simão, F. A., Waterhouse, R. M., loannidis, P., Kriventseva, E. V. \& Zdobnov, E. M. BUSCO: Assessing genome assembly and annotation completeness with single-copy orthologs.

43. Harney, E. et al. De novo assembly and annotation of the European abalone Haliotis tuberculata transcriptome. Mar. Genomics 28, 11-16 (2016). 
45. Hilgers, L., Hartmann, S., Hofreiter, M. \& von Rintelen, T. Novel genes, ancient genes, and gene co-option contributed to the genetic basis of the radula, a molluscan innovation. Mol. Biol. Evol. 35, 1638-1652 (2018).

46. Kofler, R., Pandey, R. V. \& Schlötterer, C. PoPoolation2: Identifying differentiation between populations using sequencing of pooled DNA samples (Pool-Seq). Bioinformatics 27, 3435-

47. Feder, J. L., Egan, S. P. \& Nosil, P. The genomics of speciation-with-gene-flow. Trends Genet. 28, 342-50 (2012).

48. Seehausen, O. et al. Genomics and the origin of species. Nat. Rev. Genet. 15, 176-92 (2014).

49. von Rintelen, T. \& Glaubrecht, M. New discoveries in old lakes: three new species of Tylomelania Sarasin \& Sarasin, 1897 (Gastropoda: Cerithioidea: Pachychilidae) from the Malili lake system on Sulawesi, Indonesia. J. Molluscan Stud. 69, 3-17 (2003).

50. Mack, K. L. \& Nachman, M. W. Gene regulation and speciation. Trends Genet. 33, 68-80 (2017).

51. Alvarez, M., Schrey, A. W. \& Richards, C. L. Ten years of transcriptomics in wild populations:

52. Grabherr, M. G. et al. Full-length transcriptome assembly from RNA-Seq data without a

53. Haas, B. J. et al. De novo transcript sequence reconstruction from RNA-seq using the Trinity platform for reference generation and analysis. Nat. Protoc. 8, 1494-1512 (2013).

54. Konczal, M. et al. Genomic response to selection for predatory behavior in a mammalian model of adaptive radiation. Mol. Biol. Evol. 33, 2429-2440 (2016).

55. Hughes, T. A. Regulation of gene expression by alternative untranslated regions. Trends

56. Haygood, R., Babbitt, C. C., Fedrigo, O. \& Wray, G. A. Contrasts between adaptive coding and noncoding changes during human evolution. Proc. Natl. Acad. Sci. U. S. A. 107, 7853-7857 (2010).

58. Young, M. D., Wakefield, M. J., Smyth, G. K. \& Oshlack, A. Gene ontology analysis for RNAseq: Accounting for selection bias. Genome Biol. 11, R14 (2010).

694 59. Liao, Z. et al. In-depth proteomic analysis of nacre, prism, and myostracum of Mytilus shell. J. Proteomics 122, 26-40 (2015).

696 60. Williams, S. T. Molluscan shell colour. Biol. Rev. (2016). 
697 61. Williams, S. T. et al. Colorful seashells: Identification of haem pathway genes associated with the synthesis of porphyrin shell color in marine snails. Ecol. Evol. (2017).

62. Hendry, G. A. \& Jones, O. T. Haems and chlorophylls: comparison of function and formation. J. Med. Genet. 17, 1 LP - 14 (1980).

63. Moon, S. Rho GTPase-activating proteins in cell regulation. Trends Cell Biol. 13, 13-22 (2003).

64. Redmond, L. \& Ghosh, A. The role of Notch and Rho GTPase signaling in the control of dendritic development. Curr. Opin. Neurobiol. 11, 111-117 (2001).

65. Polacheck, W. J. et al. A non-canonical Notch complex regulates adherens junctions and vascular barrier function. Nature 1-22 (2017).

66. Vo, K. et al. Targeting notch pathway enhances rapamycin antitumor activity in pancreas cancers through PTEN phosphorylation. Mol. Cancer 10, 138 (2011).

67. Zakrzewski, A. C. et al. Early divergence, broad distribution, and high diversity of animal chitin synthases. Genome Biol. Evol. 6, 316-325 (2014).

68. Lamichhaney, S. et al. Rapid hybrid speciation in Darwin's finches. Science 4593, eaao4593 (2017).

69. Lamichhaney, S. et al. A beak size locus in Darwins finches facilitated character displacement during a drought. Science 352, 470-474 (2016).

70. Chaves, J. A. et al. Genomic variation at the tips of the adaptive radiation of Darwin's finches. Mol. Ecol. 25, 5282-5295 (2016).

71. Meier, J. I. et al. Demographic modelling with whole-genome data reveals parallel origin of similar Pundamilia cichlid species after hybridization. Mol. Ecol. 123-141 (2016).

72. Enciso-Romero, J. et al. Evolution of novel mimicry rings facilitated by adaptive introgression in tropical butterflies. Mol. Ecol. 26, 5160-5172 (2017).

73. Marques, D. A., Meier, J. I. \& Seehausen, O. A combinatorial view on speciation and adaptive

74. Gazave, E. et al. Origin and evolution of the Notch signalling pathway: An overview from eukaryotic genomes. BMC Evol Biol 9, 249 (2009).

75. Richards, G. S. \& Degnan, B. M. The dawn of developmental signaling in the metazoa. Cold Spring Harb. Symp. Quant. Biol. 74, 81-90 (2009).

76. Heller, E. \& Fuchs, E. Tissue patterning and cellular mechanics. J. Cell Biol. 211, 219-231 (2015).

77. Roberts, R. B., Hu, Y., Albertson, R. C. \& Kocher, T. D. Craniofacial divergence and ongoing adaptation via the hedgehog pathway. Proc. Natl. Acad. Sci. 108, 13194-13199 (2011). 
the cichlid jaw. Proc. R. Soc. B Biol. Sci. 284, 20171018 (2017).

79. Hu, Y. \& Albertson, R. C. Hedgehog signaling mediates adaptive variation in a dynamic functional system in the cichlid feeding apparatus. Proc. Natl. Acad. Sci. 111, 8530-8534 (2014).

80. Rojas-Ríos, P., Guerrero, I. \& González-Reyes, A. Cytoneme-mediated delivery of hedgehog regulates the expression of bone morphogenetic proteins to maintain germline stem cells in Drosophila. PLoS Biol. 10, e1001298 (2012).

81. Cicconardi, F., Marcatili, P., Arthofer, W., Schlick-Steiner, B. C. \& Steiner, F. M. Positive diversifying selection is a pervasive adaptive force throughout the Drosophila radiation. Mol. Phylogenet. Evol. 112, 230-243 (2017).

82. Albertson, R. C. \& Kocher, T. D. Genetic and developmental basis of cichlid trophic diversity.

83. Albertson, R. C., Streelman, J. T. \& Kocher, T. . D. Genetic basis of adaptive shape differences in the cichlid head. J. Hered. 94, 291-301 (2003).

84. Fish, J. L. Evolvability of the vertebrate craniofacial skeleton. Semin. Cell Dev. Biol. (2017).

85. Pires-daSilva, A. \& Sommer, R. J. The evolution of signalling pathways in animal development. Nat. Rev. Genet. 4, 39-49 (2003).

86. Jiggins, C. D., Wallbank, R. W. R. \& Hanly, J. J. Waiting in the wings: What can we learn about gene co-option from the diversification of butterfly wing patterns? Philos. Trans. R. Soc. B $\mathbf{3 7 2}$, 20150485 (2016).

87. Rohlf, F. J. (Department of E. and E. S. U. of N. Y. at S. B. TpsDig2. (2006).

88. Adams, D. C. \& Otárola-Castillo, E. geomorph: an R package for the collection and analysis of geometric morphometric shape data. Methods Ecol. Evol. 4, 393-399 (2013).

89. Holznagel, W. E. A nondestructive method for cleaning gastropod radulae from frozen, alcoholfixed, or dried material. Am. Malacol. Bull. 14, 181-183 (1998).

756 90. Rasband, W. . (U. S. N. I. of H. ImageJ.

91. Sultan, M. et al. A simple strand-specific RNA-Seq library preparation protocol combining the Illumina TruSeq RNA and the dUTP methods. Biochem. Biophys. Res. Commun. 422, 643-646 (2012).

92. Joshi, N. A. \& Fass, J. N. Sickle: a sliding-window, adaptive, quality-based trimming tool for FastQ files. (2011).

93. Martin, M. Cutadapt removes adapter sequences from high-throughput sequencing reads. EMBnet. J. 17, 10-12 (2011).

764 94. Langmead, B., Trapnell, C., Pop, M. \& Salzberg, S. L. Ultrafast and memory-efficient alignment 
of short DNA sequences to the human genome. Genome Biol. 10, R25 (2009).

95. Li, B. \& Dewey, C. N. RSEM: Accurate transcript quantification from RNA-Seq data with or without a reference genome. BMC Bioinformatics 12, 323 (2011).

96. Li, W. \& Godzik, A. Cd-hit: A fast program for clustering and comparing large sets of protein or nucleotide sequences. Bioinformatics 22, 1658-1659 (2006).

97. Robinson, M. D., McCarthy, D. J. \& Smyth, G. K. edgeR: A Bioconductor package for differential expression analysis of digital gene expression data. Bioinformatics 26, 139-140

98. Li, H. et al. The sequence alignment/map format and SAMtools. Bioinformatics 25, 2078-2079 (2009).

99. Roesti, M., Salzburger, W. \& Berner, D. Uninformative polymorphisms bias genome scans for

100. Konczal, M., Koteja, P., Stuglik, M. T., Radwan, J. \& Babik, W. Accuracy of allele frequency estimation using pooled RNA-Seq. Mol. Ecol. Resour. 14, 381-392 (2014).

101. Kozak, G. M., Brennan, R. S., Berdan, E. L., Fuller, R. C. \& Whitehead, A. Functional and population genomic divergence within and between two species of killifish adapted to different osmotic niches. Evolution (N. Y). 68, 63-80 (2014).

102. Schlötterer, C., Tobler, R., Kofler, R. \& Nolte, V. Sequencing pools of individuals - mining genome-wide polymorphism data without big funding. Nat. Rev. Genet. 15, 749-763 (2014).

103. Westram, A. M. et al. Do the same genes underlie parallel phenotypic divergence in different Littorina saxatilis populations? Mol. Ecol. 23, 4603-4616 (2014).

104. Al-Tobasei, R. et al. Identification of SNPs associated with muscle yield and quality traits using allelic-imbalance analyses of pooled RNA-Seq samples in rainbow trout. BMC Genomics 18, $1-15(2017)$.

105. Supek, F., Bošnjak, M., Škunca, N. \& Šmuc, T. Revigo summarizes and visualizes long lists of gene ontology terms. PLoS One 6, (2011). 